\title{
Hotness-aware Sensor Networks
}

\author{
Dong $\mathrm{Li}^{1,3}$, Yanmin $\mathrm{Zhu}^{2,4}$, Li Cui ${ }^{1}$, Lionel M. Ni ${ }^{2}$ \\ ${ }^{1}$ Institute of Computing Technology, Chinese Academy of Sciences \\ ${ }^{2}$ Hong Kong University of Science and Technology \\ ${ }^{3}$ Graduate School of the Chinese Academy of Sciences \\ ${ }^{4}$ Imperial College London \\ \{lidong,lcui\}@ict.ac.cn,yzhu@doc.ic.ac.uk, ni@cs.ust.hk
}

\begin{abstract}
In a realistic sensor network, in particular with a nonuniform deployment, sensor nodes inevitably have varying workloads. This causes a natural problem that some sensor nodes are subject to excessive power consumption and thus become hot. These hot nodes deplete much earlier resulting in system performance degradation. This paper proposes a systematic approach to design a hotness-aware sensor network where each node is able to obtain its own hotness information. Based on these vital information, the system is able to provide various technologies to protect the critical set of hot nodes. More specifically, we design a centralized optimal algorithm to derive the precise hotness of each node. In addition, we develop a completely distributed algorithm to estimate hotness with high accuracy. An effective hotness-aware MAC is developed to offer medium access priority to the nodes with higher hotness to protect and prolong their lifetimes. It is demonstrated, through both theoretical analysis and comprehensive simulations, that our approach is valuable to improving system performance of practical sensor networks.
\end{abstract}

\section{Introduction}

Realistic deployment of of wireless sensor networks (WSNs) usually results in non-uniform distribution of sensor nodes due to physical constraints such as terrain variation. Some nodes are likely to have excessive workloads, e.g., forwarding data, and to consume more power. An example is shown in Fig. 1. A node is defined hotter if its power consumption rate is higher. Hot nodes have significant impact on the overall system performance since they will deplete much earlier, which may lead to the emergence of blind areas and disconnection of the network. Thus, it is of paramount importance to build a sensor network where each node awares its hotness. With these hotness information, the system can adopt effective mechanisms to protect

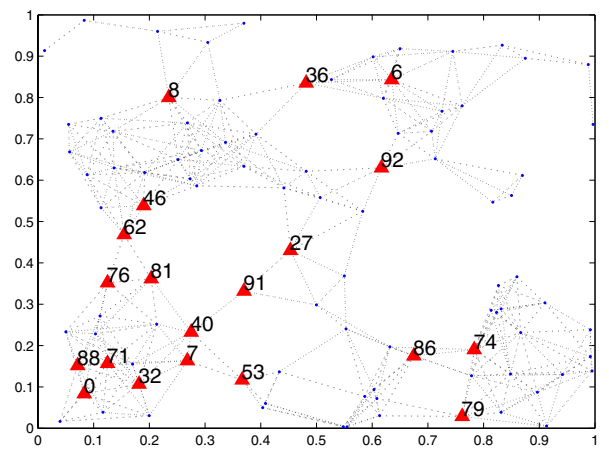

\section{Figure 1. The distribution of hot nodes, where the red triangles represent the top 20 with the heaviest traffic load. Node 0 is the sink.}

hot nodes and hence maintain high performance.

In large-scale WSNs, the existence of hot nodes is inevitable. Several key facts account for this. Firstly, the many-to-one traffic pattern in many applications usually results in unbalanced load distributions. Secondly, the random deployment may lead to non-uniform network topology. It is unavoidable that some critical nodes in such network (e.g., node 36 in Fig.1) become hot even if loadbalancing protocols are applied.

Regarding the issue of hot nodes, intensive research efforts have been made in power-aware routing [2,8], clustering structures $[12,18]$, congestion control algorithms [6] and hybrid MAC protocols [11]. However, most of the prior approaches did not consider the problem rooted at the nonuniform distribution of sensor nodes, and hence failed to address this issue completely [1]. The researchers [1] have studied the hot nodes problem near the sink and proposed Funneling-MAC based on a sink-centered control mechanism. However, the problem still exist at places where the node density is relatively low in a non-uniform topology. For example, nodes 36 and 92 in Fig. 1 are more than 5 
hops away from the sink and the sink-centered MAC control is insufficient for them. In contrast to most existing studies, we consider it is inappropriate to treat all sensor nodes equally. Hot nodes should be protected better so that their limited resources can be utilized for the best purpose. For example, to consume less power, hot nodes may be offered higher priority to access the channel.

In this paper, we propose a systematic approach to design a hotness-aware sensor network. We adopt a unified framework to estimate the hotness of all nodes, which takes into account both the intrinsic topology and the fact that various routing algorithms can be employed for data communication. We design an effective MAC protocol that exploits hotness information and protect hot nodes from consuming excess power on competing to access the channel to prolong their lifetimes. To the best of our knowledge, this is the first paper concerning itself with this crucial problem in realistic sensor networks.

There are several technical challenges. Firstly, it is difficult to estimate the hotness of a sensor node since it depends on many factors, such as different routing schemes adopted, the distribution of source nodes and the data generation rates. Secondly, it is infeasible for a node to compute extensively due to its resource limitation, therefore the efficiency of the distributed algorithms must be considered. The original contributions of this work are summarized as follows.

- We investigate the hotness issue thoroughly and study the implication of network topology on node hotness. A novel framework with a new hotness metric, hot potential, is proposed for the hotness estimation.

- We design a centralized algorithm HPA to compute node hotness precisely. A distributed algorithm DHPA is also devised for the resource-constrained nodes particularly. We analyze the convergence step and communication complexity of DHPA and show the high accuracy of DHPA by numerical simulation.

- We develop a novel MAC protocol, HP-MAC, which guarantees hotter nodes higher priority to access the channel to achieve significant energy saving.

The rest of the paper is organized as follows. Section 2 describes system model and analysis framework. The centralized algorithm and the distributed algorithm are presented in Section 3 and 4, respectively. Section 5 elaborates on the design and implementation of HP-MAC. Section 6 presents simulation results. Section 7 discusses related work. We conclude our work in Section 8.

\section{System Model and Problem Formulation}

In this section we describe the system model and formulate the problem of hotness estimation in a given deployment of sensor network.

\subsection{Network Model}

Sensor nodes are considered static with the same amount of initial energy. There is one sink node collecting data. The deployment of sensor networks is non-uniform, which reflects real-world applications.

A sensor network is defined as a directed graph $G=$ $(V, E)$, where $V$ is the set of sensor nodes and $E$ is the set of directed links $(u, v)$, where $u, v \in V$. Each link $(u, v) \in E$ has a nonnegative routing metric $c(u, v) \geq 0$. If $(u, v) \notin E$, we assume that $c(u, v)=\infty$. The sink node is denoted as $t$. For every node $s \in\{V-t\}$, there is at least one path from $s$ to $t$, denoted as $s \rightarrow v^{*} \rightarrow t$. The source nodes generate data periodically. We define the set of source nodes as source group, denoted as $S$.

Routing scheme has high impact on node hotness. To be energy-efficient, single-path routing has been widely utilized for data collection. Here we assume single-path routing algorithms are employed. A simple path is denoted as:

$$
p=\left\langle v_{0}, v_{1}, \ldots, v_{i}, \ldots, v_{k}\right\rangle, v_{i} \in V, v_{0}=s, v_{k}=t
$$

The cost of the path is defined as $c(p)=\sum_{i=1}^{k} c\left(v_{i-1}, v_{i}\right)$. The set of all paths from $s$ to $t$ is denoted as $E_{s, t}$. We sort these paths in the order of increasing cost. The rank of a path $p$ is denoted as $r_{p}$. Then the routing model can be defined by the general routing abstraction function as follows.

Definition 1. (General Routing Abstract Function): The general routing abstract function $f\left(r_{p}\right)$ defines the probability of path $p$ being chosen as a route from all candidates in $E_{s, t}$, where $\sum_{p \in E_{s, t}} f\left(r_{p}\right)=1$.

Based on the routing model, we can see that for the shortest path routing algorithm $f(1)=1, f\left(r_{p}\right)=0$ if $r_{p}>1$.

\subsection{Analytical Model}

We propose an analytical model to facilitate the computation and analysis of node hotness. The basic idea of our analysis is that given a network $G$, a source group $S$ and the routing abstract function $f\left(r_{p}\right)$, node hotness can be computed. For a hot node, we believe that it is more likely included in routes from source nodes to the sink. For a specific sensor node, we list all paths traversing this node from the source group to the sink. According to the routing model, we can infer the probability of this node to be selected to forward data for a source node. Then we aggregate the probabilities of all the paths through this node. It is apparent that the aggregated probability is a good indicator of the node hotness.

Formally, we have basic concepts defined as follows:

Definition 2. (Hot Degree): The hot degree of node $v, v \in$ $V$, is the traffic per unit time at node $v$, denoted as $d_{v}$. 
Definition 3. (Potential Route Set): The potential route set $R_{v}$ of node $v$ contains the simple paths traversing node $v$ from the source group to this sink, formally $R_{v}=\{p: p \in$ $\left.E_{s, t}, \forall s \in S, v \in p\right\}$.

Definition 4. (Hot Potential): The hot potential of node $v$, $h_{v}$, is denoted as:

$$
h_{v}=\sum_{p \in R_{v}} f\left(r_{p}\right), v \in V .
$$

It is extremely difficult to compute the exact hot potential of a node, as shown by the following theorem.

Theorem 1. Computing the hot potential of node $v$ by finding the potential route set of this node is computationally infeasible, given network $G$, source group $S$ and general routing abstract function $f\left(r_{p}\right)$.

Proof. Unlike an NP problem stated in a decision form, computing hot potential is a counting problem associated with the decision problem. This kind of problem is known as \#P problem in [13]. The proof has two parts. Firstly, given $E_{s, t}, s \in S$, to find $R_{v}$ is polynomial in $\left|E_{s, t}\right||S|$. The sum operation of $f\left(r_{p}\right)$ is also polynomial in $\left|R_{v}\right|$. Secondly, we need to enumerate all possible paths between $s$ and $t$. A simpler version of this subproblem, counting the number of simple paths between $s$ and $t$ in a directed graph, has been proved to be \#P-Complete [13]. Therefore, computing the hot potential contains a subproblem no easier than a \#P-Complete problem. It is computationally infeasible.

\section{Centralized Optimal Algorithm}

In this section, we devise a centralized algorithm to estimate node hot potentials. We first relax the general routing abstract model to the zero-tailed routing abstract function to avoid the intractable complexity of enumerating all possible routes. Then we present the centralized algorithm HPA and analyze its complexity.

\subsection{Relaxed Routing Model}

Typically, an effective routing algorithm intends to select the paths with minimal cost, therefore we can only consider the best paths. For this purpose, we relax the general routing abstract function as follows:

Definition 5. (Zero-tailed Routing Abstract Function): The zero-tailed routing abstract function $f_{K}\left(r_{p}\right)$ defines the probability of path p being selected as a route:

$$
f_{K}\left(r_{p}\right)= \begin{cases}\frac{1}{K}, & r_{p} \leq K \\ 0, & r_{p}>K,\end{cases}
$$

where $K$ is the number of candidate routes that the routing alogrithm considers.

In this paper, we do not fix the routing metric, as it depends on the design of routing algorithm.

\subsection{Centralized Hot Potential Algorithm}

The centralized hot potential algorithm (HPA) reflects the definition of hot potential. The algorithm essentially has two key steps.

Step 1: For each source node $s \in S$, we employ Yen's algorithm [17] to find top $K$ shortest-paths from $s$ to $t$. We utilize the ranking of paths found in Yen's algorithm to set $r_{p}$. All the possible routes from the source nodes to the sink are stored in $E_{S, t}$.

Step 2: For each node $v \in V-t$, we search for $R_{v}$ from the paths in set $E_{S, t}$. After that, we compute $h_{v}$ as the sum of values of $f_{K}\left(r_{p}\right)$ of these paths.

Our technical report [7] gives the pseudo code of HPA.

\subsection{Algorithm Analysis}

The complexity of HPA is mainly determined by the network size and the routing scheme. We divide routing algorithms into two classes. One class employs rational routing metric and selects the best routing path that satisfies the predefined threshold, such as MintRout [15]. This class adopts a constant $K$ (e.g., 1) in the zero-tailed routing abstract function. With these algorithms, HPA can achieve the optimal solution in polynomial time as indicated by theorem 2 .

Theorem 2. Given network $G$ and function $f_{K}\left(r_{p}\right)$, in the worst case, the time complexity of HPA is $O\left(K^{2}(m+\right.$ $n \log n+1)$ ), where $n=|V|$ and $m=|E|$.

The other class chooses routes randomly from all possible routes every time the node needs to transmit packets, e.g., Gossip routing [4]. With this class of routing schemes, the computability of HPA depends upon how $K$ is decided. When $\mathrm{K}$ is not a constant, we have the following theorem:

Theorem 3. Given a sensor network $G=(V, E)$ and function $f_{K}\left(r_{p}\right)$, if $K$ is linear to the number of $s-t$ paths (e.g., $\left.K=\alpha\left|E_{s, t}\right|\right)$, the time complexity of HPA is $O\left(\alpha n^{n+2}(m+\right.$ $n \log n+1)$ ), where $n=|V|$ and $m=|E|$.

The proof of these two theorems can be found in [7].

It is apparent that HPA is computationally inefficient for large-scale sensor networks.

\section{Distributed Algorithm}

To deal with the high computational cost of HPA, a distributed algorithm, called DHPA, is proposed which allows each node to estimate its hotness in terms of hot potential locally and interact with neighbors.

\subsection{Distributed Hot Potential Algorithm}

The distributed hot potential algorithm (DHPA) should estimate hot potentials as accurately as possible. Meanwhile, we should minimize the computation and communication costs. The key to compute the hot potential is to 


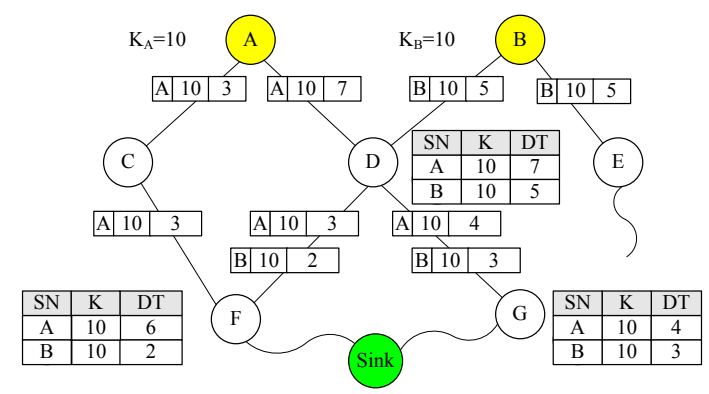

Figure 2. A probing process in DHPA

identify the top $K$ shortest paths from each source node to the sink. Before delving into the details, we first illustrate the basic idea. DHPA will be executed in the initialization phase since the hot nodes should be protected as early as possible. At the beginning, each source node initiates a probing process, in which expedition packets are sent out to discover the top $K$ shortest paths. The nodes along the discovered paths are therefore informed, and are able to gradually update their hot potentials. Each node obtains its final hotness estimate when all the probing processes terminate.

A probing process is illustrated in Fig. 2. It starts from source node $A$ and $B$. Suppose that $A$ has 10 paths to find. $A$ dispatches the tasks of finding 3 paths and finding 7 paths to $C$ and $D$, respectively. Upon receiving the expedition packet, $D$ knows that 7 paths pass it from $A$ to the sink and $A$ has 10 paths to find. Thus, relative to source node $A$, the hotness of $D$ is set to 0.7 . $D$ again dispatches the task received from $A$ to its neighbors $F$ and $G$. The dispatching operation repeats until an expedition packet reaches the sink. A node may receive expedition packets containing tasks originated from different source nodes. For example, node $D$ receives expedition packets from both $A$ and $B$. At each node, a table is maintained to keep the records of different source nodes. Each record has three fields: the source node ID (SN), the number of paths the source node needs to find $(K)$, the number of paths the node should find for this source node (DT). The record $\langle S N, K, D T\rangle$ is also used in expedition packets.

When deciding to dispatch tasks among its neighbors, a node faces several problems. The first is which neighbors to choose to continue the probing process. The second is how many paths to dispatch to a neighboring node. The third problem is that the number of paths should always be an integer, but not a fraction. We propose a greedy policy to make decisions. A node only chooses the neighbors with minimum routing cost to continue the probing process (we use downstream neighbors for short to denote these neighbors. In addition, the upstream neighbors of a node are those neighbors whose routing cost is larger than its own cost). The path-finding task will be first dispatched to the node with the smallest routing cost and the number

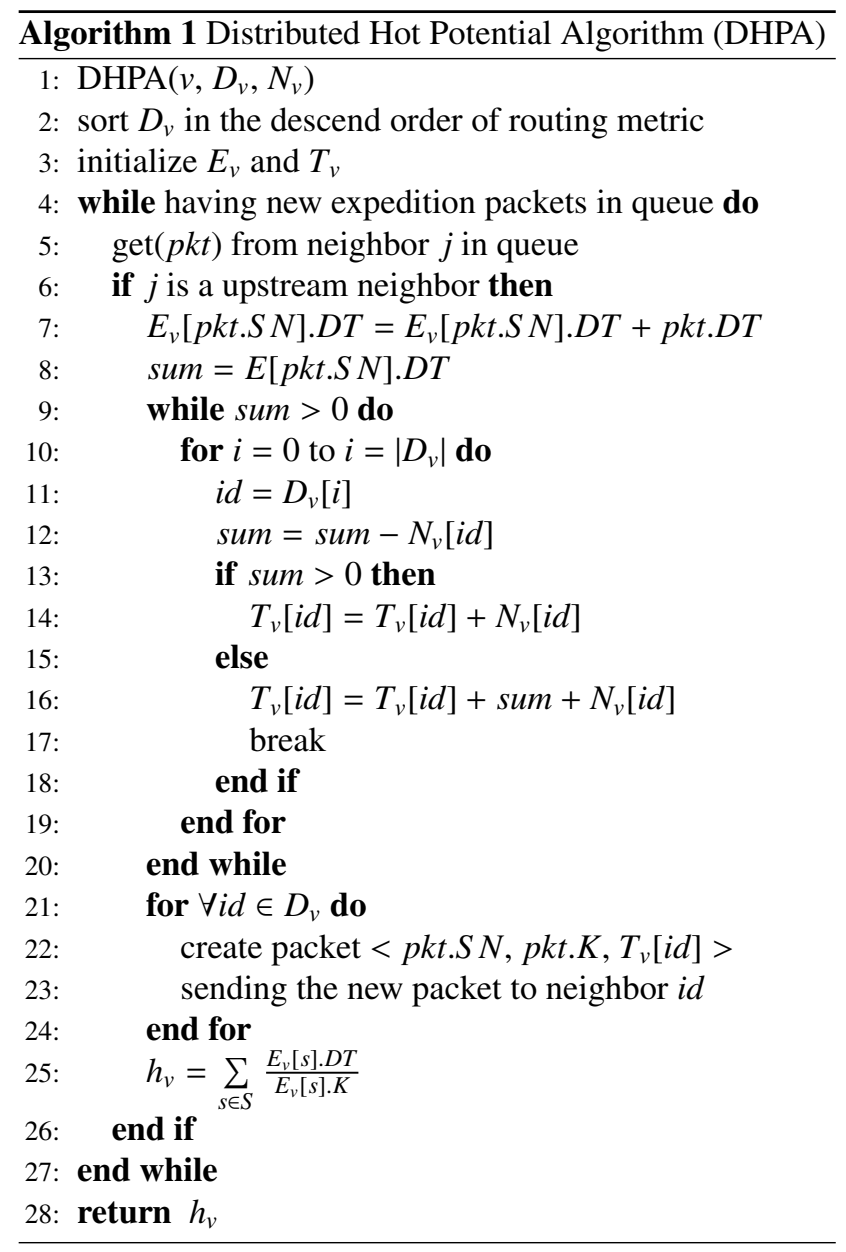

of paths of this subtask is decided by the number of downstream neighbors this node has. Then, the path-finding task is dispatched to the node with the second smallest routing metric. This process repeats until all the paths needed to be found are dispatched out. Formally, we give the pseudo code in Algorithm 1 where node $v$ maintains: a hashtable $E_{v}$ to keep the records for source nodes; $D_{v}$ is an array containing IDs of the downstream neighbors of node $v$; Hashtable $T_{v}$ stores the number of paths node $v$ plans to dispatch to each downstream neighbor; Hashtable $N_{v}$ stores the number of downstream neighbors of the downstream neighbors of node $v$. All the hashtables take the node ID as the key to the corresponding value.

\subsection{Algorithm Properties}

The convergence and communication cost properties of this algorithm can be shown in the following two theorems.

Theorem 4. DHPA converges in $|S|(|V|-|S|-2)$ steps, in the worst case.

Proof. DHPA running on node $v$ converges when all its upstream neighbors have no new expedition packet to send. 
Considering the worst case of topology that every upstream neighbor of node $v$ is used as relay node by all the source nodes, DHPA needs to update local hashtable $E_{v}$ for $|S|\left|U_{v}\right|$ times where $U_{v}$ is the set of upstream neighbors of node $v$. Then we consider the maximum value of $|S|\left|U_{v}\right|$. Since $|S|>0$ and $|S|$ is a constant, we can derive that the maximum $\left|U_{v}\right|$ brings the maximum value of update times. Node $v$ and the sink is excluded from $U_{v}$. The upstream neighbor of node $v$ can not be a source node in the worst case because a source node will not send expedition packets to the other upstream neighbors of node $v$ if it connects with node $v$, therefore the maximum value of $\left|U_{v}\right|$ is $|V|-|S|-2$. In the worst case, DHPA converges in $|S|(|V|-|S|-2)$ steps.

Theorem 5. The communication cost of DHPA is $O\left(|S| K_{\max }\left(D_{\max }-1\right)\right)$ in the worst case, where $K_{\max }$ is the maximum value of $K$ used by the source nodes. $D_{\max }$ is the hops of the longest shortest path in the network.

Proof. The probing process of DHPA starts from each source node independently. We first consider the worst case of single source nodes, in which the source node has the longest shortest path to the sink. Suppose this path has $D_{\max }$ hops. The source node needs to find at most $K_{\max }$ paths of $D_{\max }$ hops. In this process, the number of expedition packets is $K_{\max }\left(D_{\max }-1\right)$ because the sink is excluded from the probing process. Extending this conclusion to the case of multiple source nodes, the worst case is that the source nodes are all $D_{\max }$ hops away from the sink and they all have $K_{\max }$ shortest paths to find. The total communication complexity is $O\left(|S| K_{\max }\left(D_{\max }-1\right)\right)$.

\subsection{Numerical Results}

For a non-uniform topology, DHPA can achieve high accuracy in hotness estimation, which is validated by simulations. For the topology in Fig. 1 where 100 nodes are deployed in a $1000 m \times 1000 m$ rectangular area, we simulate that all the nodes report data to the sink at the data rate of $1 \mathrm{pps}$. The real hot degree is measured when the shortest path routing is employed. The hot potential is estimated by HPA and DHPA. As shown in Fig. 3(a), the cumulative distributions of the hotness are very close. To compare the accuracy of node hotness information, we define the hotness ranking error as follows.

$$
A_{v}=\frac{r_{h_{v}}-r_{d_{v}}}{|V|}
$$

where $r_{h_{v}}$ is the rank of $h_{v}$ among all the sensor nodes in terms of hot potential, and $r_{d_{v}}$ is the rank of $d_{v}$ among all the sensor nodes in terms of hot degree. In Fig. 3(b), most of the hotness ranking errors estimated by HPA and DHPA are distributed between $[-0.3,0.3]$.

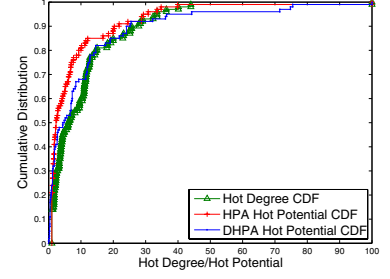

(a) Hotness (hot degree/hot potential) cumulative distribution.

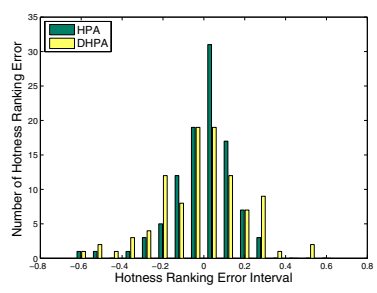

(b) The distribution of Hotness ranking error of HPA.
Figure 3. Accuracy comparison

\section{Hotness-aware MAC}

Hot nodes are usually critical and therefore need to be well protected in the sensor network. In this section, we propose a hotness-aware MAC protocol, called HP-MAC, to protect hot nodes through giving them higher priority in channel accessing. The importance of hotness information is meanwhile demonstrated by the usefulness of this novel MAC protocol. HP-MAC employs hot potential information to predict node hotness. Its unique feature is that hot nodes can be protected at the early stage after the network deployment and prevent congestions around them. HPMAC enables hotter nodes to temporarily control the forwarding operations of its neighbors and thus to work more energy efficiently.

HP-MAC is implemented based on B-MAC and adopts the invitation mechanism by giving privileges to the hotter node to manage the schedule of the channel access around it. A hot node use an invitation beacon to invite its upstream neighbors to transmit sequentially, and to inform its downstream neighbors to receive packets in a given period of time. According to the node hot potentials, HP-MAC integrates the multiple random medium accesses around the hot node into an ordered schedule. By this means, the cost of competing for the channel at the hot node is saved and the loss rate around it can also be reduced.

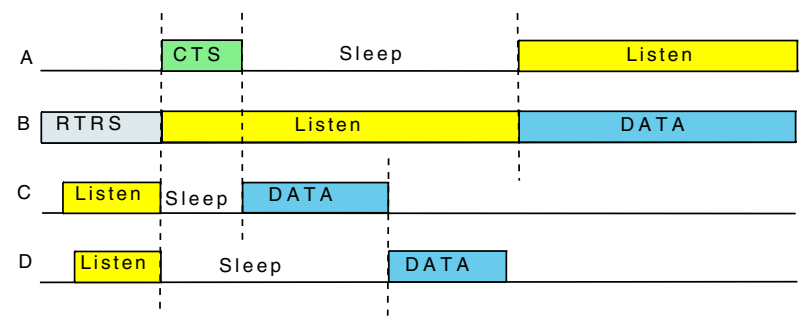

Figure 4. A forwarding process of HP-MAC

A full sequence of packet transmission of HP-MAC is illustrated in Fig. 4. The sequence is driven by the node with higher hot potential, such as node $B$. Node $B$ decides the transmissions of its upstream neighbors with lower hot potentials, such as $C$ and $D$. Node B broadcasts a RTRS 
(Ready to Receive and Send) beacon indicating that it is ready to receive packets from upstream neighbors and will transmit these packets immediately to node $A$ who is a downstream neighbor of $B$ with lower hot potential. We call the whole process as a forwarding process. A RTRS beacon contains two kinds of information. One is the transmission sequence of the transmitters. The other is the number of packets that can be sent by each transmitter. The RTRS beacon can be viewed as a temporal TDMA schedule for a single forwarding process. When $A$ receives a RTRS, it sends out a CTS (Clear to Send) and then go to sleep. The length of sleep time is decided by the total number of transmission packets in the RTRS.

HP-MAC exploits the hot potential information computed by the DHPA algorithm. The action of a node is determined by the following rule: A node can only schedule the sending or receiving operations of its one-hop neighbors with lower hot potential. With the local hot potential information within one hop, a node knows whether it has the right to control the receiving of its downstream neighbors. A node keeps the packets received in queue and waits for RTRS beacon if all its downstream neighbors have larger hot potential. A node will ignore the RTRS from its neighbors with lower hot potential.

We discuss several implementation issues of HP-MAC on control overhead reduction, configuration of RTRS packet and dealing with dynamic traffic. Due to space limitation, we do not present algorithm analysis here. Our technical report [7] provides more detailed information.

\section{Performance Evaluation}

The goal of our evaluation is to validate our design and study the performance of HP-MAC. We compare our approach with two state-of-the-art schemes, Funneling-MAC [1] and B-MAC [10]. In simulation experiments, we use the fundamental power-consumption datasheet of MICA2 node [3]. We focus on four metrics: (1) node lifetime is the time duration until the node is depleted. If the node is still alive when the sink receives the last data packet, its lifetime is the time until the last data packet is received; (2) loss rate is the ratio of the number of packets received at the sink to the number of packets generated by all source nodes; (3) throughput is the amount of bits of received data packets by the sink in unit time and (4) packet delivery delay is the time duration from the time when the packet is generated to the time when it reaches the sink.

The important simulation parameters are listed in Table 1. The settings of Funneling-MAC and B-MAC are configured to default values as specified in $[1,10]$.

\subsection{Node Lifetime Distribution}

To illustrate HP-MAC's ability to alleviate the hot nodes problem, we perform the simulations using the topology
Table 1. Simulation Parameters

\begin{tabular}{ll}
\hline Simulation Parameter & Value \\
\hline field area & $1000 \mathrm{~m} \times 1000 \mathrm{~m}$ \\
communication range & $200 \mathrm{~m}$ \\
bandwidth & $19.2 \mathrm{kbps}$ \\
number of nodes & 100 \\
packet length & $36 \mathrm{bytes}$ \\
initial energy & $4 \mathrm{~J}$ \\
\hline
\end{tabular}

shown in Fig. 1. All nodes report to the sink at the data rate of 1pps. We first compare the cumulative distributions of the average node lifetime, as shown in Fig. 5. HP-MAC achieves increased longevity of node lifetimes. The curve of HP-MAC has fewer points falling in interval $[0,0.1]$ on axis Y. The lifetimes of all the red nodes in Fig. 1 are extended, which prolongs the lifetimes of their upstream neighbors. We use a histogram view to show the number of nodes falling into different lifetime intervals. Fig. 6 indicates that HP-MAC achieves more balanced power consumption among the nodes due to the fair channel allocation based on node hotness. B-MAC and Funneling-MAC show a big gap between the last three adjacent lifetime intervals, while HP-MAC has a smaller difference gap.

\subsection{Loss Rate}

In the experiments on loss rate, we vary the data rate and the source group size, separately. The topology and the source nodes are randomly generated. Figure. 7 shows that the loss rate of HP-MAC is consistently lower at various data rates when the source group size is 16 . The channel contention around hot nodes is alleviated by invitation of more packets to transmit in one RTRS. This mechanism keeps the curve of loss rate rising slowly with increasing data rates. The loss rate of HP-MAC is also much lower at various source group sizes at the fixed data rate of $1 \mathrm{pps}$. The results shown in Fig. 8 indicate that HP-MAC works efficiently at a medium load. HP-MAC assumes that every node with a non-zero hot potential relays traffic, which leads to redundant RTRS beacons with a small source group size. When the source group size increases, more RTRS beacons trigger real packet forwarding. Therefore, the loss rate exhibits a stable state with different traffic loads.

\subsection{Throughput}

We first evaluate the throughput of HP-MAC with different data rates. We randomly choose 16 source nodes out of all nodes. The throughput results are shown in Fig. 9. HP-MAC consistently achieves higher throughput than BMAC and Funneling-MAC for data rates from 0.2 to $4 \mathrm{pps}$. The throughput curve of HP-MAC exhibits a descending trend when the data rate is higher than 4pps because HPMAC is forced to invite more packets in a single RTRS in heavy traffic scenarios. This increases the waiting time of 


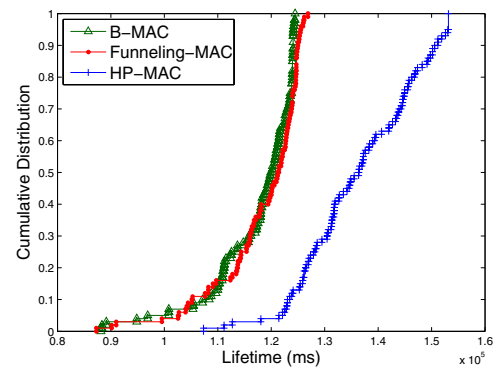

Figure 5. Lifetime cumulative distribution of nodes using the topology in Fig.1.

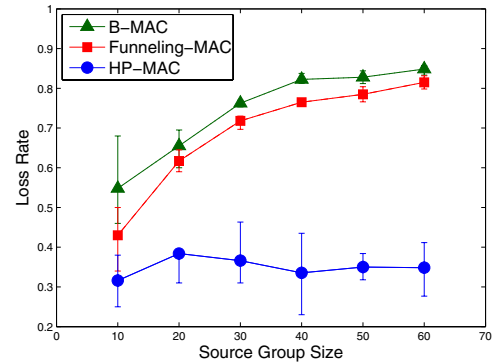

Figure 8. Loss rate with varying source group sizes in random topologies.

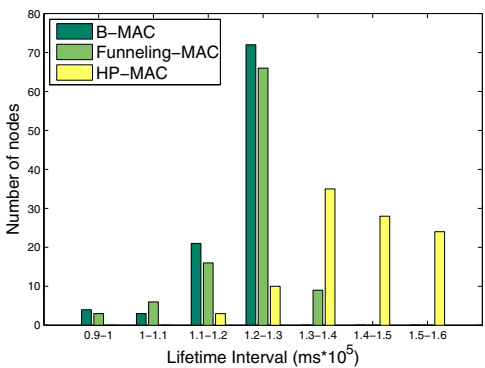

Figure 6. The number of nodes which fall in continuous lifetime intervals.

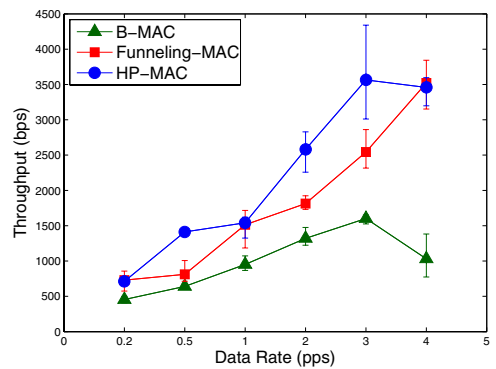

Figure 9. Throughput with varying data rates in random topologies.

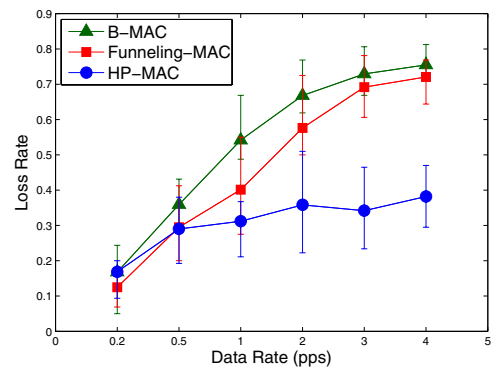

Figure 7. Loss rate with varying data rates in random topologies.

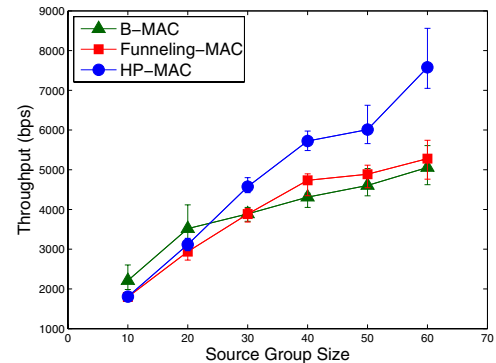

Figure 10. Throughput with varying source group sizes in random topologies. a packet in queue as the relay node waits more time for its next sending slot. The results of throughput with various source group sizes at data rate of 1pps are shown in Fig. 10. When the source group size is small, B-MAC achieves a little higher throughput because B-MAC has no control overhead. HP-MAC achieves better performance than B-MAC and Funneling-MAC when there are more source nodes. The invitation mechanism of HP-MAC works more efficiently than the TDMA mechanism of funneling-MAC at a medium load level, since the traffic monitoring method takes more time to measure node traffic.

\subsection{Packet Delivery Delay}

HP-MAC shows a negative impact on packet delivery delay. Hotter nodes control the channel access around them, which inevitably slows down the relay action of neighbors. To investigate the impact of this mechanism itself (duty cycle configuration, overload traffic and large network diameter also affect the delay heavily), we run the simulations at a medium traffic load (e.g., 1pps) and a medium-sized network (e.g., 30nodes) with the full duty cycle. The topology is randomly generated with 10 source nodes. Through the simulations for the network of 6-hop diameter, the average packet delay is $274.24 \mathrm{~ms}$. HP-MAC experiences longer delivery delay compared with B-MAC and Funneling-MAC.
HP-MAC prolongs the lifetime of hot nodes remarkably, achieves consistently lower loss rate and provides better throughput. The modest cost of longer packet delivery delay is acceptable for most applications, such as environment monitoring and target surveillance.

\section{Related Work}

There have been significant interests in power-aware designs $[2,8,9,16]$. The residual battery status and communication energy consumption are major concerns in these works. Optimization techniques are usually used to study energy conservation in routing and topology management. Energy-efficient clustering algorithms $[12,18]$ provide an alternative way to avoid hot nodes problem. These algorithms utilize two techniques: cluster head rotating and balancing traffic load among various clusters. These techniques suffer excessive control overhead. Furthermore, they cannot prevent critical nodes from extensive data forwarding in non-uniform networks. The removal of these nodes will significantly decrease the vertex connectivity of the topology graph.

More recently, hybrid scheduling methods $[1,5,11]$ have been proposed in the MAC layer to mitigate the hot nodes problem. Normal hybrid MAC $[5,11]$ protocols maintain the absolute fairness in contention among the nodes. Actu- 
ally, hot nodes need higher priority to achieve higher power efficiency. Funneling-MAC [1] is designed for the funneling effect which can be regarded as a kind of hot nodes problem occurring around the sink. The sink node manage the TDMA schedule in Funneling-MAC. As a result, there is a maximum depth in hops from the sink to the boundary of TDMA region due to the maximum radio transmission range. This depth limits the scale of the network FunnelingMAC can serve. For areas with relatively low sensor density in a large-scale network, Funneling-MAC is of little help. Our approach works for non-uniform networks and achieves efficient hotness estimation with high scalability.

One may argue that measuring-based "hot" metrics, e.g. load traffic [1], transmit queue occupancy [14], residual battery capability [8] and average number of noise backoff [11], are also good indicators of hotness. However, we have identified the serious limitation: those metrics are time lagged. Nodes is already in "hot" state when the problem is detected. Usually, it comes at the cost of high loss rate and longer packet delay for a node to recover from the hot state. In contrast, hot potential is prediction-based. A node can be protected from the very beginning of a network.

\section{Conclusions and Future Work}

In this paper, we have presented a systematic approach to designing a hotness-aware sensor network. The framework of hot potential is valuable in predicting node hotness information. The decentralized algorithm is practical even for large-scale sensor networks. It can estimate node hotness accurately, which is demonstrated via comparison against the optimal centralized algorithm. The hotness information is very useful as it provides construction guidance for many technologies to protect critical hot nodes. To demonstrate the use of hotness information, we have designed the HP-MAC, in which hotter nodes are given higher priority in accessing the wireless media. As a result, the precious energy of hot nodes are effectively conserved. In addition, the natural congestion around hot nodes are well managed. Simulation experiments show that HP-MAC consistently achieves better performance than the state-of-the-art schemes in terms of power balance, loss rate and throughput. HP-MAC achieves these performance gains at a slight cost of packet delivery delay. This is acceptable, however, for most realistic applications of sensor networks.

In the future, we will further study the distributed algorithm for multiple sinks. Moreover, more practical use of the hot potential information will be explored. Finally, a prototype will be constructed to implement our approach and to study its performance in a real network setting.

\section{Acknowledgment}

We thank the invaluable comments from the reviewers. This work is co-sponsored by National Basic Research Pro- gram of China grant 2006CB303000, UK EPSRC grant EP/C547586/1, NSFC grants 60533110 and 60572060, High-Tech R\&D Program of China grant 2006AA01Z215 and CAS Innovation Project KGCX2-YW-110-3.

\section{References}

[1] G.-S. Ahn, E. Miluzzo, A. T. Campbell, S. G. Hong, and F. Cuomo. Funneling-mac: A localized, sink-oriented mac for boosting fidelity in sensor networks. In Proceedings of ACM SenSys, 2006.

[2] J.-H. Chang and L. Tassiulas. Maximum lifetime routing in wireless sensor networks. IEEE/ACM Transactions on Networking, 12:609-619, 2004.

[3] Crossbow Technology. Mica2 datasheet. http://www. xbow.com/.

[4] Z. J. Haas, J. Y. Halpern, and L. Li. Gossip-based ad hoc routing. IEEE/ACM Transactions on Networking, 14:479491, 2006.

[5] B. Hohlt, L. Doherty, and E. Brewer. Flexible power scheduling for sensor networks. In Proceedings of ACM/IEEE IPSN, 2004.

[6] B. Hull, K.J., and H. Balakrishnan. Mitigating congestion in wireless sensor networks. In Proceedings of ACM SenSys, 2004.

[7] D. Li, Y. M. Zhu, L. Cui, and L. M. Ni. Hotness-aware sensor network. Technical Report ICT_WSN_TR20080301, ICT CAS, 2008.

[8] Q. Li, J. Aslam, and D. Rus. Online power aware routing in wireless ad hoc network. In Proceedings of ACM MobiCom, 2001.

[9] X. Li, W. Song, and W. Wang. A unified energy efficient topology for unicast and broadcast. In Proceedings of ACM MobiCom, 2005.

[10] J. Polastre, J. Hill, and D. Culler. Versatile low power media access for wireless sensor network. In Proceedings of ACM SenSys'04, 2004.

[11] I. Rhee, A. Warrier, M. Aia, and J. Min. Z-mac: a hybrid mac for wireless sesnor networks. In Proceedings of ACM SenSys, 2005.

[12] T. Shu, M. krunz, and S. Vrudhula. Power balanced coverage-time optimization for clustered wireless sensor networks. In Proceedings of ACM MobiHoc, 2005.

[13] L. Valiant. The complexity of enumeration and reliability problems. SIAM Journal on Computing, 8:410-421, 1979.

[14] C.-Y. Wan, S. E. Eisenman, and A. T. Campbell. Coda: congestion detection and avoidance in sensor networks. In Proceedings of ACM SenSys, 2003.

[15] A. Woo and D. Culler. Taming the underlying challenges of reliable multihop routing in sensor networks. In Proceedings of ACM SenSys, 2003.

[16] G. Xing, C. Lu, Y. Zhang, Q. Huang, and R. Pless. Minimum power configuration in wireless sensor networks. In Proceedings of ACM MobiHoc, 2005.

[17] J. Yen. Finding the $k$ shortest loopless paths in a network. Management science, 17:712-716, 1972.

[18] O. Younis and S. Fahmy. Heed: A hybrid, energy-efficient distributed clustering approach for ad hoc sensor networks. IEEE Transactions on Mobile Computing, 3:366-379, 2004. 